\title{
METABOLIC INTEGRATION: BEYOND THE BUILDING BLOCKS
}

\author{
Machado, M.D.V.' ${ }^{1}$ Alves-Oliveira, M.F.'; Sodré, C.L. ${ }^{1}$ \\ ${ }^{1}$ Departamento de Biologia Celular e Molecular, Instituto de Biologia, \\ Universidade Federal Fluminense, Rio de Janeiro, Brazil; ${ }^{2}$ Centro \\ Universitário de Volta Redonda, Rio de Janeiro, Brazil
}

The theme "metabolic Integration" discussed during Biochemistry classes is considered by many students a complex issue. It could be due to their difficulty in understanding that the metabolic pathways are not isolated reactions, but a completely interdependent system finely regulated. Given this reality, a didactic game was developed. The main objective was to challenge students to understand the metabolism integration, through a playful, interactive and dynamic way. The class was divided into groups and to each group was given a set of parts that represented an important pathway of energetic metabolism. The aim of each group was to complete the metabolic process assigned to them. However, during the assembly, they realized that was always lacking some part of the puzzle and that the game only succeeds if all the groups exchange parts with each other. After that, the pieces came together in order to assemble all the processes in an integrated way. The game was organized into two situations: metabolic reactions that occur in the fasted state and reactions of the fed state. When the groups realized they needed to join themselves to complete the processes, they also had to get into a consensus that the "body" in which the reactions were happening, was in a fasted state or not, because the pieces didn't match each other if both metabolic states were being assembled at the same time. It is not suitable to the organism performs the reactions of antagonistic states at the same time/or at the same velocity. Along the schema assembly, key points were didactically marked in some pieces with colors and warnings. The proposal was to open a discussion after assembling of the parts. The game was applied to students at the first year of medicine school and had a great acceptance.

Key words: metabolism, integration, game. 